\title{
Scanning the Auroral Skies: The Athabasca University Robotic Telescope
}

\author{
Ian Schofield ${ }^{1 *}$, Martin Connors ${ }^{1,2}$, Phil Langill ${ }^{2,3}$, Stefan Cartledge ${ }^{4}$
}

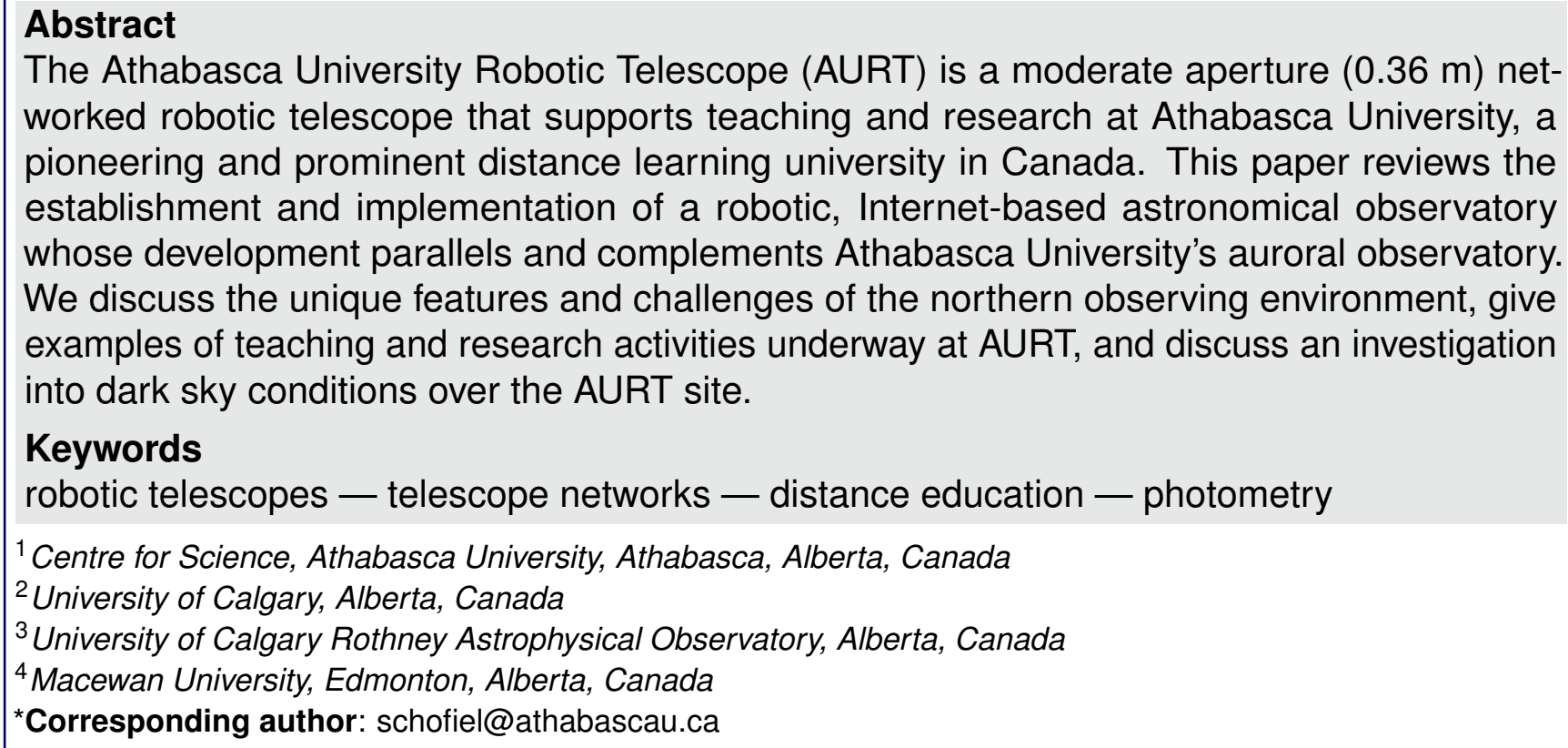

\section{Introduction}

The aurora borealis is a fixture over the Canadian prairies, particularly during the long dark winter months given their proximity to the northern auroral zone. This roughly 10 degree wide band encircling the north magnetic pole sweeps across Alaska and the northern half western Canada. Athabasca University, located in the town of Athabasca, Alberta, is situated in a sub-auroral location at 54.6 degrees north, slightly south of the auroral oval. A critical requirement for selecting an auroral observation site suitable for research-quality all-sky observations are exceptionally dark skies (see figure 1). Population density drops off as one goes north in Alberta, so that light pollution could also be expected to diminish. Athabasca University is a distance education institution, unusually among high-enrolment universities (ca. 40,000 students), located in a small town setting. Its campus grounds in the late 1990s and early 2000s were sufficiently dark to support auroral observation, which led to the establishment of the Athabasca University Geophysical Observatory (AUGO) in 2002. The dark sky requirements for auroral all-sky observation are ideal for an astronomical observatory, so three years later, the Athabasca University Robotic Telescope (AURT) was built, co-located on the AUGO site.

\section{Creation of AURT}

The primary intended role for AURT was to serve as an automated telescope for performing sky surveys to search for near-earth objects and exoplanets. Ideally, AURT would be linked to a software pipeline that would produce a steady stream of research data to assist researchers in identifying new objects. AURT could carve out a niche role in observing high latitude objects given that there were 


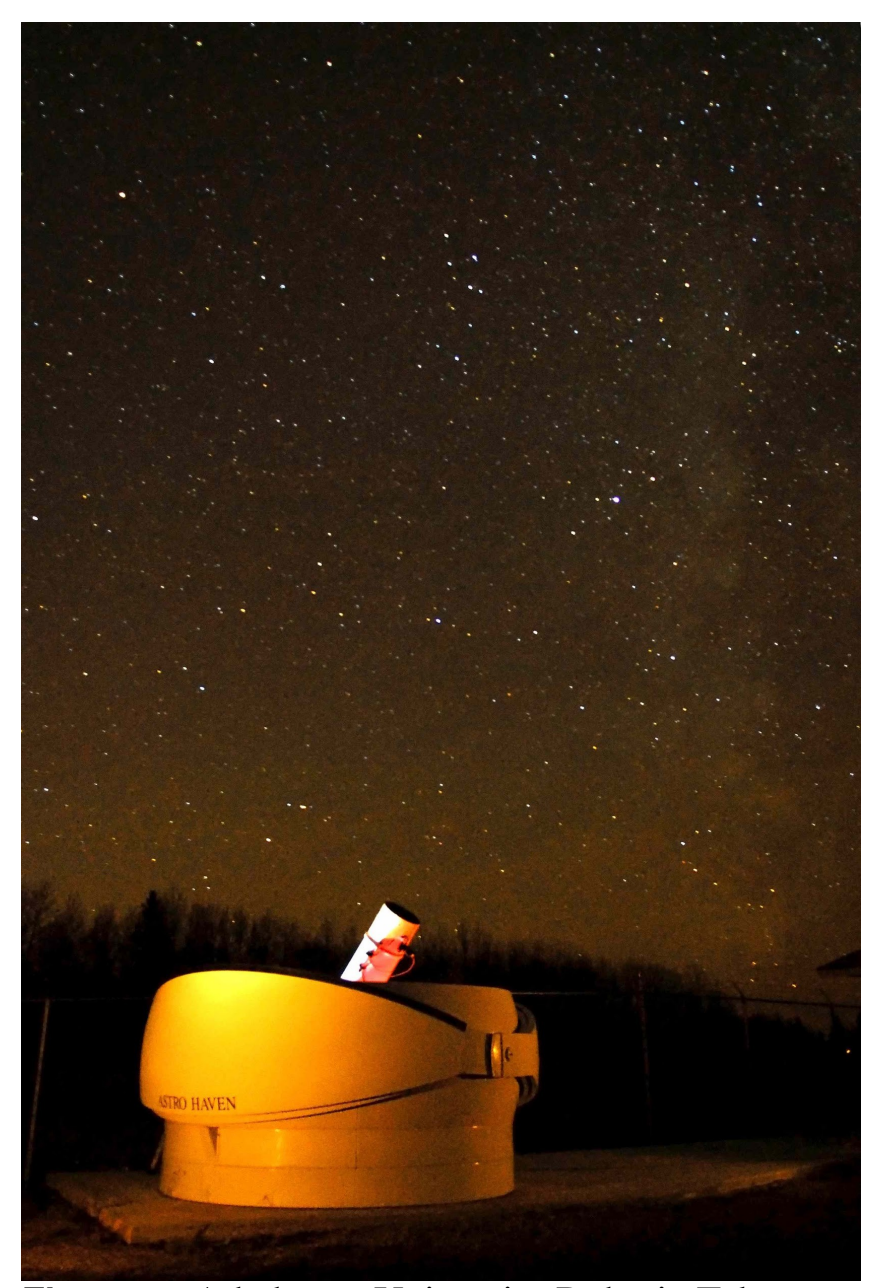

Figure 1. Athabasca University Robotic Telescope (AURT) as it appeared in 2006, set against the southern sky prominently featuring the Milky Way. Pictured is the original custom-built 14-inch Newtonian telescope. At this time, the AURT site was located in a secluded area of the Athabasca University campus, on the edge of the town of Athabasca, Alberta. It was indeed sufficiently dark to support auroral observations at the neighboring Athabasca University Geophysical Observatory (AUGO). Photo by Mathieu Meurant.

few research-oriented telescopes in high latitude areas conducting surveys of the northern skies. Although the density of observable objects is low, in principle there are interesting targets to be observed or discovered at high ecliptic latitudes. Ironically, the Earth Trojan class of objects nominally able to be discovered by such surveys was found by other means (Connors et al. 2011, 2014). Further, by taking advantage of its northernly Alberta location, AURT could become a test center for northernbased, remote, autonomous robotic telescopes, ultimately leading to the laying of the groundwork for meter-class Arctic telescopes in the future (Martin and Connors 2008). Recognizing Athabasca University's role as a leader in distance education and its mandate to deliver science education at a distance, a robotic telescope can also serve as a powerful teaching tool supporting research-based astronomy courses.

AURT reached operational status as a robotic telescope in 2006. It consisted of a f/5.6 14-inch Newtonian reflector, coupled with a German- equatorial fork mount. Both were custom engineered by an advanced amateur telescope builder associated with the Edmonton Centre of the Royal Astronomy Society of Canada (RASC). The telescope was housed in a 12-foot diameter motorized clamshell dome, built by Astrohaven Enterprises. AURT was upgraded in 2013 to address lingering technical problems with the telescope mount that were preventing reliable operation. AURT in its current state is pictured in figure 2.

The most prominent change to AURT after the 2013 upgrade was the switch to a networked, queuebased observation system, made possible by joining the Skynet Robotic Telescope Network. Operated by the University of North Carolina Chapel Hill (UNC), Skynet is a worldwide network of modestaperture telescopes whose fundamental task is to facilitate rapid target acquisition of gamma ray bursts (GRB) (Reichart 2006). Given the rarity of GRBs, the telescope network can also be put to use serving observing requests using a queue-based observation scheduler via web-based user interface. Skynet offers a networked instrument control and data management infrastructure that allows simple, efficient and secure access to telescope observing time. It serves thousands of users worldwide and includes students, astronomy enthusiasts and professional researchers. Smith and Caton provide a detailed account of their experience adapting their legacy robotic telescope to function as a networked Skynet telescope and provide many details on the design and operation of the Skynet telescope net- 


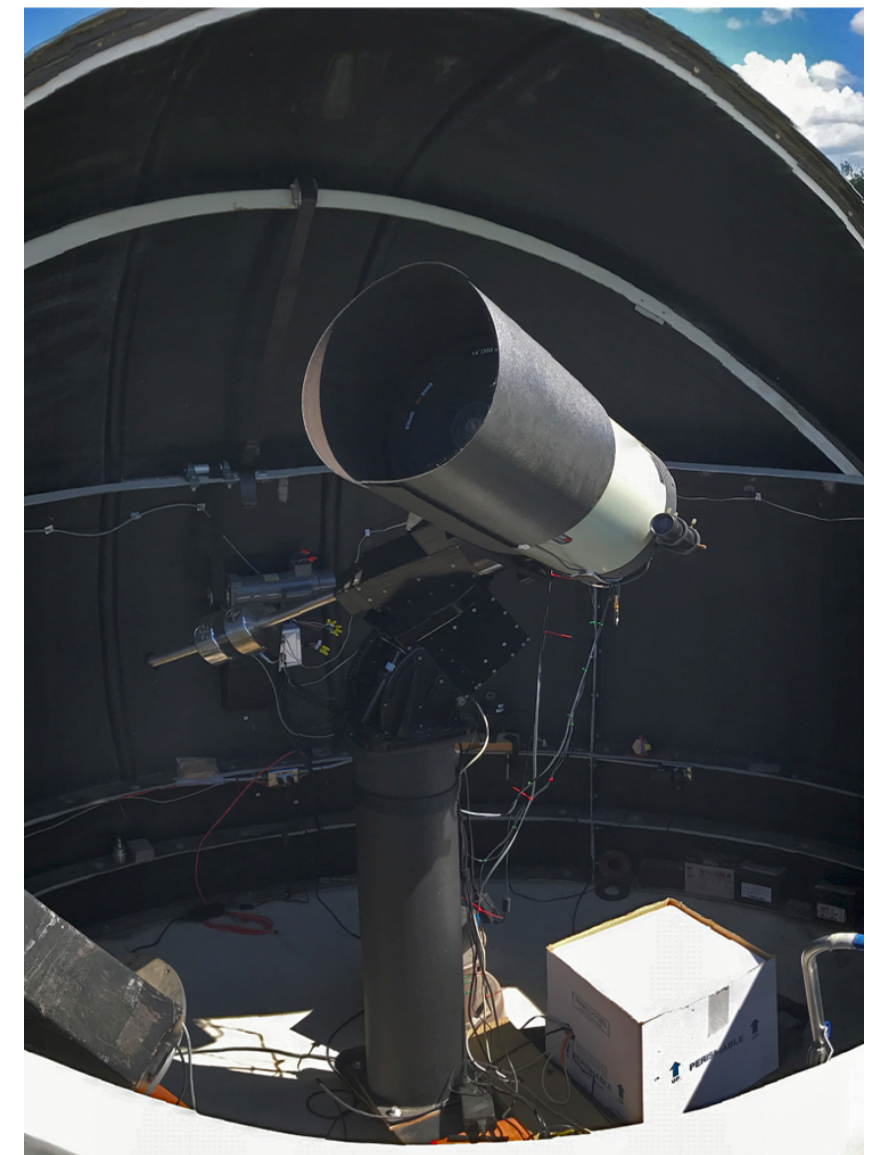

Figure 2. AURT in 2018, picturing the west side of the Astrohaven dome lowered. AURT's Celestron Edge 1400HD $0.36 \mathrm{~m} \mathrm{f} / 10$

Schmidt-Cassegrain telescope, mounted on a Software Bisque Paramount ME robotic German equatorial mount, forms the core components of the AURT. AURT has been a member of the Skynet telescope network since 2014 and is one of a growing number of Internet-based moderate-aperture telescopes. Photo: Ian Schofield

work (Smith et al. 2016; Caton 2018).

\section{Operating Environment}

The observing environment in Athabasca allows for extended observing periods during the winter months due to its high geodetic latitude. For example, in December, astronomical darkness can persist as long as 14 hours. Provided there are clear skies, long periods of darkness are advantageous for performing long photometric observations, such as in collecting light curves. The tradeoff to long wintertime observing is proportionally short dark sky periods during summer months. AURT experiences no astronomical twilight between May 8 and August 4. The Athabasca region occasionally witnesses dramatic auroral activity such as pictured in figure 3. The northern half of Western Canada attracts auroral researchers and tourists as it is an accessible region in the northern hemisphere from which to see the northern lights. Despite the breathtaking beauty of the aurora borealis, it is a form of natural light pollution and does affect astronomical observations.

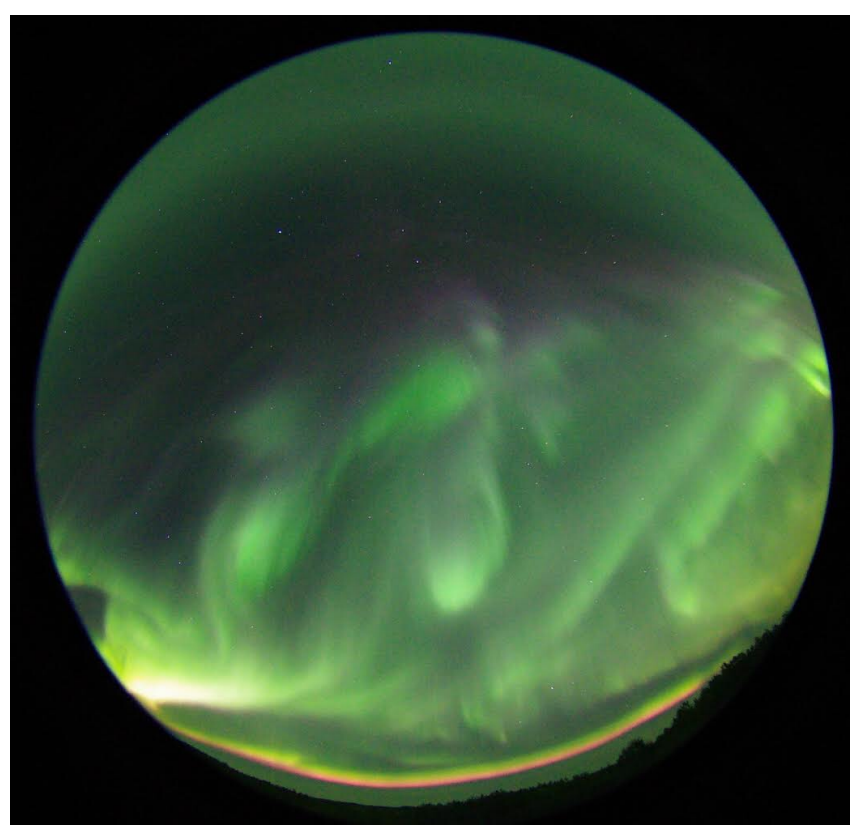

Figure 3. Astronomical observations can be affected by bright auroral displays, such as the one pictured, which occurred on September 16, 2017. This all-sky auroral photograph shows the skies above the Athabasca University Geospace Observatory, situated $25 \mathrm{~km}$ southwest of the town of Athabasca. The bottom of the image shows the northern horizon. Photo: Athabasca University Geospace Observatory.

Athabasca's temperate continental climate (Köppen class Dfb), bordering on subartcic, means that in the prime winter season, low temperatures, exacerbated by wind, affect nearly all the components of the observatory. As temperatures dip below -30 degrees $\mathrm{C}$, mechanical systems gradually lose reliability: solenoid switches start to stick, camera filter movement becomes labored or seizes, and the 
mount begins to generate errors. As recommended by the manufacturer, we swapped out the default worm gear lubricant and replaced it with low temperature grease. As a rule, we halt operations with AURT when temperatures dip below -30 degrees C. However, most affected, from our experience, is the dome.

Snow in northern Alberta is usually relatively light, but it must be swept off the dome after a fresh snowfall. Unlike tracking domes or roll-off roofs, clamshell domes like the Astrohaven have the potential to dump accumulated snow onto the telescope below when opened. When snow resting the dome melts under sunny conditions, water drippings freeze and accumulate on the sides of the domes, which can jam the dome when opening. Furthermore, ice film can develop along the seam between the two outer shells, effectively welding them shut. Damage to the fiberglass dome shells has happened when the frozen or jammed outer shells come apart and come crashing down. We run a heating cord along the seam of one of the outer shells to prevent ice film formation, and keep the dome cleared of snow to prevent snow melt from occurring.

\section{Applications}

\section{Teaching}

Astronomy distance education courses have been offered at Athabasca University since 1987 and have all contained computer-based instructional content that represented the state of the art at the time (Connors 2003). Presently, Athabasca University has two senior-level project-based astronomy courses (Connors et al. 2019), Astronomy 495 and 496, that offer students the option to collect data using AURT. When the student begins the course, he or she will draft a learning contract that outlines the objectives of the study. The plan, upon approval by the supervising instructor, will detail what kind of data is required and how it will be gathered. This may include telescope observation, but other types of instrumentation, such as tracking digital cameras, have also been used in course laboratory work.

In the case of a telescope-based project, AURT's telescope operator engages with the student by setting up a Skynet account, providing operating instructions, and answering questions through email. Students are responsible for performing their own data reduction using tools recommended by their supervisor. AURT is usually involved in one student research project per observing season that runs from September through March. Although we try to start student projects as early as possible, poor weather and mechanical failures can slow down or derail student lab work. In the event that observations cannot proceed within the confines of the semester, we have worked out sharing arrangements with our Skynet partners to acquire telescope time.

\section{Collaboration}

Skynet has allowed us to enter into collaborative research and teaching arrangements with other universities and organizations. We have shared telescope time via Skynet with the U.S. 4-H Skynet Junior Skynet Scholar program (Childers et al. 2015), a program that engages middle school youth to explore the universe using robotic telescopes and analyze their data. Participating students have published variable star observations using data from AURT.

Starting in 2015, we entered into a teaching collaboration with MacEwan University that has enabled three of MacEwan's junior and senior level astronomy courses to use AURT for conducting course lab work and student-led observing projects. In 2017, MacEwan University's third-year planetary systems course used AURT to perform photometric light curve measurements of exoplanets. Students were asked to confirm attributes of known exoplanets using AURT by observing their transits. Students selected targets of interest, planned the observations, then loaded them into the Skynet observation queue through the classes' Skynet account that was set up for the course. Once the observations were completed and data downloaded, students analyzed the resulting light curves and reported on the observed planetary characteristics, comparing their results to those reported in the literature. Example data appear in figure 4. 


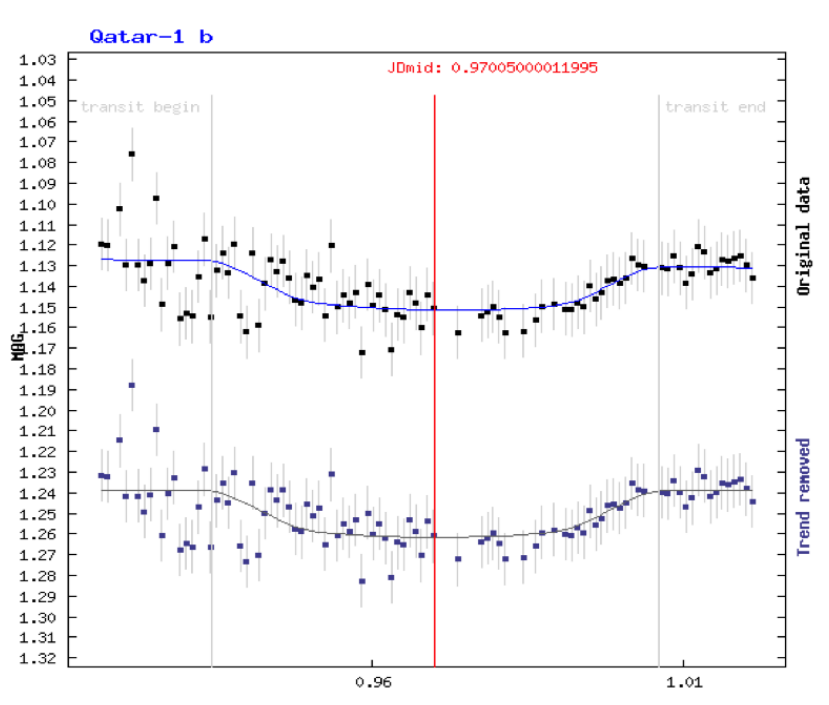

Figure 4. An example exoplanet transit observation of Qatar-1 b, taken from data gathered in the Winter of 2017 by MacEwan University student Jared Fairbanks for the PHYS 324 Origins of Planetary Systems course. This light curve, plotted from data collected by AURT, shows the raw and fitted light curve at the top. Below appears the same photometric data, with linear and quadratic trends arising from sky brightness and airmass removed. In total, 6 students used AURT to generate light curves from multiple exoplanet systems in order to confirm their attributes.

\section{AlgolCam}

As an alternative to telescope-based observations, we have had success using robotic tracking digital cameras fitted with consumer-grade zoom lenses to produce photometric light curve measurements. The system, similar in form and function as the Panoptes survey camera (Guyon et al. 2014), is called Algolcam (Connors et al. 2016). Using a $50-200 \mathrm{~mm} \mathrm{f} / 4.5 \mathrm{zoom}$ lens, set to $85 \mathrm{~mm}$ focal length, Algolcam is capable of imaging a $10 \times 15-$ degree field of view and capturing objects as faint as 12 th magnitude with a 30 -second exposure under optimal dark sky conditions. The current Algolcam design, shown in figure 5, operates under an auroral observation dome at our current auroral observatory, the Athabasca University Geospace Observatory (AUGSO). A new Algolcam design, currently in development, will be weatherproofed for outdoor operation and not require an enclosing structure. Several of our students have used Algolcam for collecting exoplanet light curves in support of their senior-level astronomy course work and have reduced their data using Muniwin for photometric image analysis, and astrometry.net (Lang et al. 2010) for field cataloging. Once Algolcam moves out of the experimental phase, we anticipate it will be an effective low-cost teaching tool, with applications in automated sky monitoring.

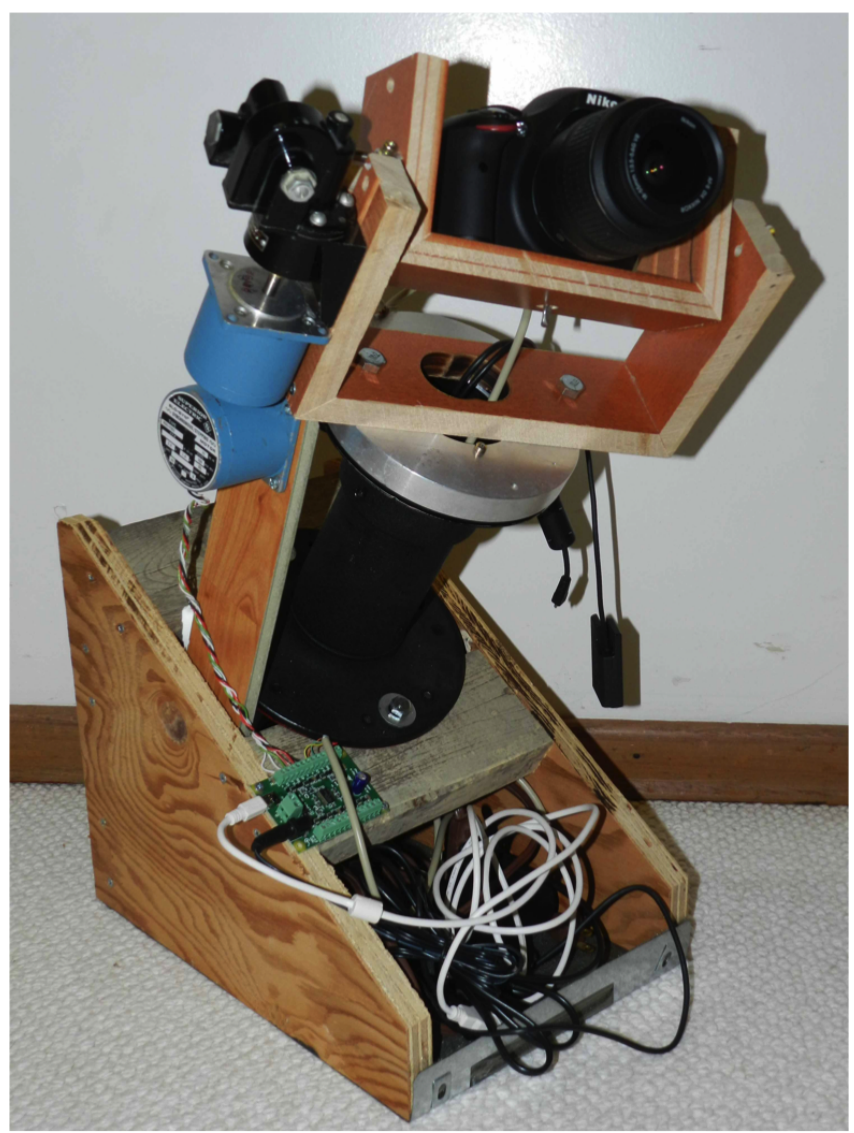

Figure 5. The first version of Algolcam, a tracking DSLR camera fitted with telephoto lens, used for performing photometric measurements on variable stars, with applications in low cost sky surveys, sky monitoring and teaching. Photo: Martin Connors

\section{Dark Sky Assessment}

New building development near the Athabasca University campus began to degrade dark sky conditions at the AUGO site as early as the late 2000s. H-beta narrowband optical measurements of proton precipitation aurora (Sakaguchi et al. 2015), 
which are highly susceptible to light pollution from street and architectural lighting, were becoming increasingly difficult to perform. This set into motion the decision to relocate auroral research outside of the town of Athabasca and establish a new auroral observatory removed from the town's light pollution footprint. The Athabasca University Geospace Observatory (AUGSO) was opened in 2012 in a dark sky location $25 \mathrm{~km}$ southwest of Athabasca. AUGSO hosts multispectral auroral optical, radio and magnetic instrumentation operated by Canadian and international partners. In November 2016, University of North Carolina installed a robotic telescope at the AUGSO site. The 16-inch RicheyChretien telescope, originally used in the UNC PROMPT cluster in Chile, went online in January 2019.

Simultaneous sky brightness measurements at the AUGO and the AUGSO sites were initiated in October 2017 in order to determine if development, which has continued unabated since 2010, is affecting our ability to view faint objects with AURT. To quantitatively measure sky brightness, we turned to the Unihedron Sky Quality Meter (SQM), which is commonly used in the amateur astronomy community (Langill and George 2017). The SQM measures sky brightness in terms of visual magnitudes per square arcsecond from a patch of sky spanning 20 degrees. The SQM's peak sensitivity, dictated by the transmittance of its internal filter, spans the visible spectrum from 300 and $700 \mathrm{~nm}$, peaking around $540 \mathrm{~nm}$. Pierantonio Cinzano's technical report on the Unihedron SQM (Cinzano 2005) provides a comprehensive analysis of the SQM device. Simply speaking, an SQM reading tells you the maximum magnitude object (meaning faintest object) you are capable of observing under the given sky conditions: the higher the visual magnitude, the darker the skies are. An exceptionally clear dark sky suitable for astronomical observing is equal to a SQM reading of 21.6, which is equivalent to a Natural Sky Unit (NSU) of 1. This unit, first described by Christopher Kyba in 2015, was based on sky brightness measurements made at Kitt Peak Observatory using a Unihedron SQM (Kyba et al. 2015).
We placed a Unihedron SQM-LE meter at the AUGO observatory, located in Athabasca, and another at the AUGSO, our current auroral observatory and dark sky site. The SQM devices were installed pointing towards zenith under the observatory's auroral observation skylights, which are composed of spectrally transparent (from IR to UV) GS2458 acrylic material acrylic material. Both devices are queried every 60 seconds by software that gathers and archives the data. By examining the SQM measurements over an extended period, we expected to see a gradual increase in the brightness of the sky over the Athabasca site. A similar study was conducted by the University of Calgary's Rothney Astrophysical Observatory (RAO) in 2016 (Langill and George 2017). Like ourselves, RAO faces similar challenges with deteriorating dark sky conditions, since we are both located on the edge of expanding urban development.

Looking for gradual increase in sky brightening in the data has proven to be difficult, due to numerous factors in play: sky cloudiness, phases of the moon, the aurora borealis, and possible darkening due to smoke particulates from summer forest fires. Similar to Langill's study, which saw the overall sky brightness over RAO between 2012 and 2015 remain relatively stable (which they attribute to effective light pollution bylaws), we did not see any detectable increase in sky brightness over the span of the data set. Figure 6 summarizes our SQM measurements taken at both sites from mid October 2017 to November 2018. The red trace shows data from AUGO (Athabasca), while the black trace depicts data from AUGSO (rural Athabasca County).

Some interesting patterns appear in the SQM data. By following the time series month by month, one can pick out the lunar cycle as represented by the monthly rise and fall of the sky brightness. The gradual widening and narrowing of the daily sky brightness curve clearly show the long nights of winter and the short nights of summer. In dark, moonless conditions, the sky brightness curve from AUGSO, being a dark-sky rural location, will appear tall and flat-topped. Similarly, we see a similar curve from AUGO, but a little less tall. In the case of cloudy conditions, however, the sky in town is 


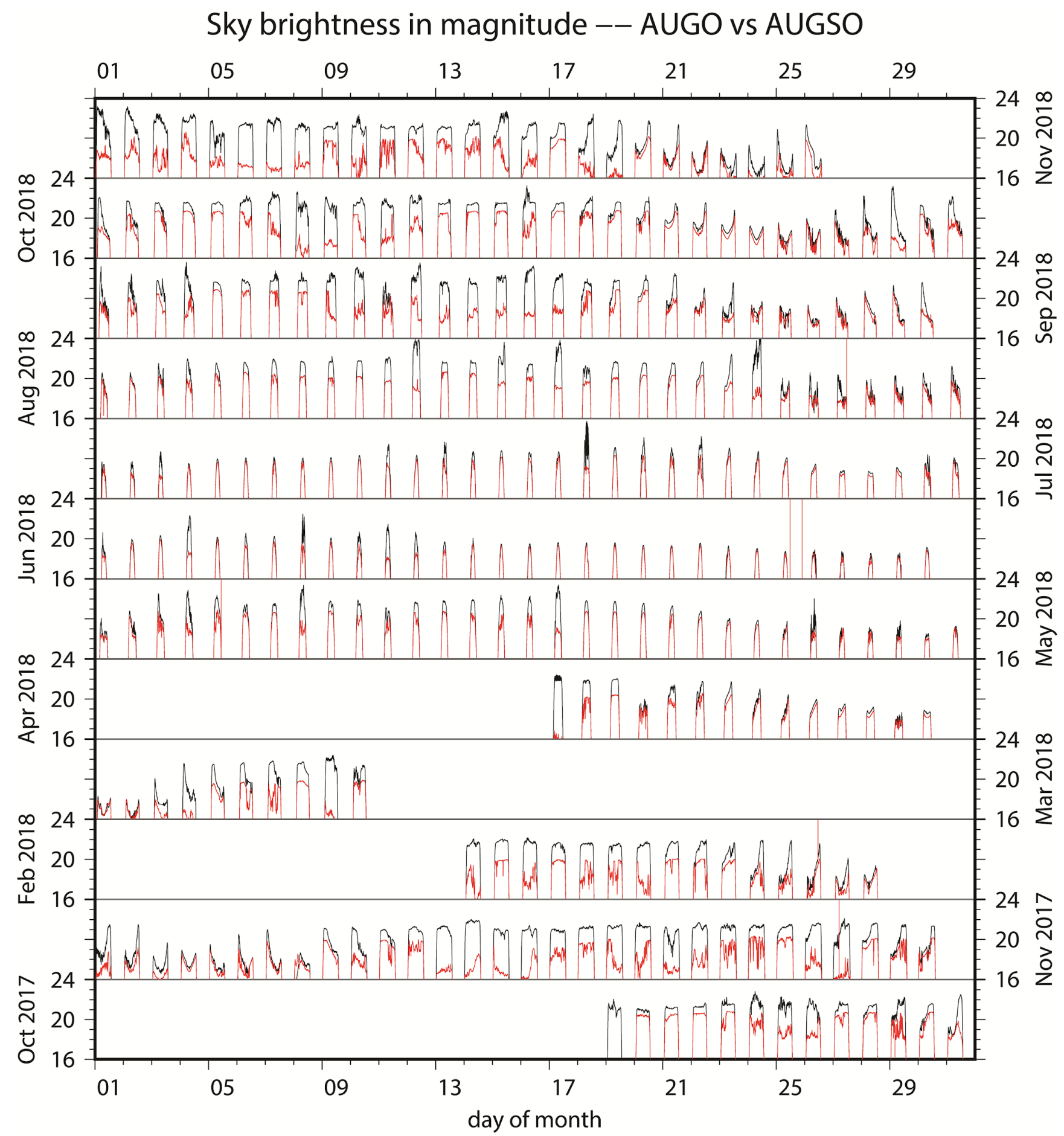

Figure 6. SQM measurements at AUGO / AURT site (in red) overlaid with SQM readings at the AUGSO site (in black). A clear offset between the data point to a difference in sky brightness magnitude between the AURT site in Athabasca, Alberta and the darker AUGSO site, located $25 \mathrm{~km}$ southwest of the town of Athabasca.

significantly brighter due to urban lighting reflecting off the clouds. On cloudy nights, skies are extremely dark in the country, while pale gray in the light polluted urban areas. The rising and setting of the moon chisels out a rounded bite off the top of the tooth-like sky brightness curve. 
It was necessary to identify a handful of nights where the sky was simultaneously dark and clear over AURT and AUGSO. To confirm that the sky was truly dark and clear (which generally should apply to both sites, since they're only $25 \mathrm{~km}$ apart), we consulted imagery taken by our Auroral all-sky imager (ASI), a Princeton Scientific ProEM EMCCD camera. A second all-sky camera, a Pentax APS-C format digital SLR, provides back up imagery where gaps exist in the EMCCD record. Additional confirmation on sky conditions was provided by Nagoya University's Canadian-based OMTI imager, based at AUGSO. ASI imagery from May 2018 show some exceptionally dark nights that were cloud free. The darkness may be due to the lack of red auroral emissions $(630 \mathrm{~nm})$, which fall within the SQM's range of spectral sensitivity. These emissions can be detected in auroral all-sky imagery, but their study is outside the scope of this paper. When they are not present, or if they do not affect astronomical imaging, the AUGSO site may at times be even darker than our averaging indicates.

There are certainly sources of systematic error, which we glossed over when doing this study. Namely, we did not measure the offset between the two units. We also did not consider the opacity of the domes, and the effect of aging of the SQM's bandpass filters. As the SQM's internal filter ages it becomes increasingly opaque, which biases the data towards higher than actual SQM sky brightness measurements. Langill's 3-year study accounted for filter degradation and reports a filter darkening rate of 0.0015 magnitude / square arcsecond / month (Langill and George 2017). We began our study using factory-new SQM units, and with only 13 months of usage, we expect their filters will be close to factory specifications. However, as we continue to gather data, we will factor in the effect of filter aging.

We identified 15 nights containing periods of exceptional dark sky conditions: free of cloud cover, moon or active aurora. We determined the average SQM sky brightness that fell on these days, within the optimal dark sky periods. The sky brightness averages, appearing in figure 7 as horizontal traces, point to a full magnitude difference in sky brightness between the rural AUGSO site and the near-urban AUGO / AURT site. The best dark sky conditions at AUGSO averaged around 21.6 visual magnitudes / square arcsecond (incredibly, as good as found at Kitt Peak), and 20.6 at AURT, on the edge of the town of Athabasca.

The SQM data set shows pronounced dark periods on August 12, 15 and 17 in 2018 that may be due to peak smoke conditions that occurred at around the same time. PM2.5 air pollution measurements over south Edmonton peaked in a natural pollution event due to wildfires several hundred $\mathrm{km}$ to the west during this period. PM2.5 measurements describe the quantity of fine particulate matter such as smoke in the atmosphere. These peaks in airborne particulates point to the heavy wildfire smoke that blanketed Alberta at this time. This interesting correlation between air pollution and sky darkening demonstrates that the SQM can be applied to atmospheric environmental monitoring. Remarkably, on some days the smoke was so dense that the SQM meters recorded it in daytime, although at magnitude levels lower than those shown in Figure 6.

\section{Summary}

AURT continues to serve as Athabasca University's astronomical teaching and research observatory and has found a role in providing student-led data gathering for intermediate and advanced undergraduate astronomy students. It may form a valuable resource for students in a proposed distance education M.Sc. program in Science. Since joining Skynet in 2013, AURT has been used by national and international observers and has a unique role as Skynet's most northernly observatory. As such, AURT fills an important niche in that it is capable of viewing objects only accessible at high latitudes and can take advantage of long observation periods during winter months. The development of AURT has paralleled and complemented Athabasca University's development of auroral observatories and has certainly benefited from the advanced infrastructure developed for its original and current auroral research facilities. We continue to use AURT for 


\section{SQM values AUGSO vs AUGO 2017-2018}

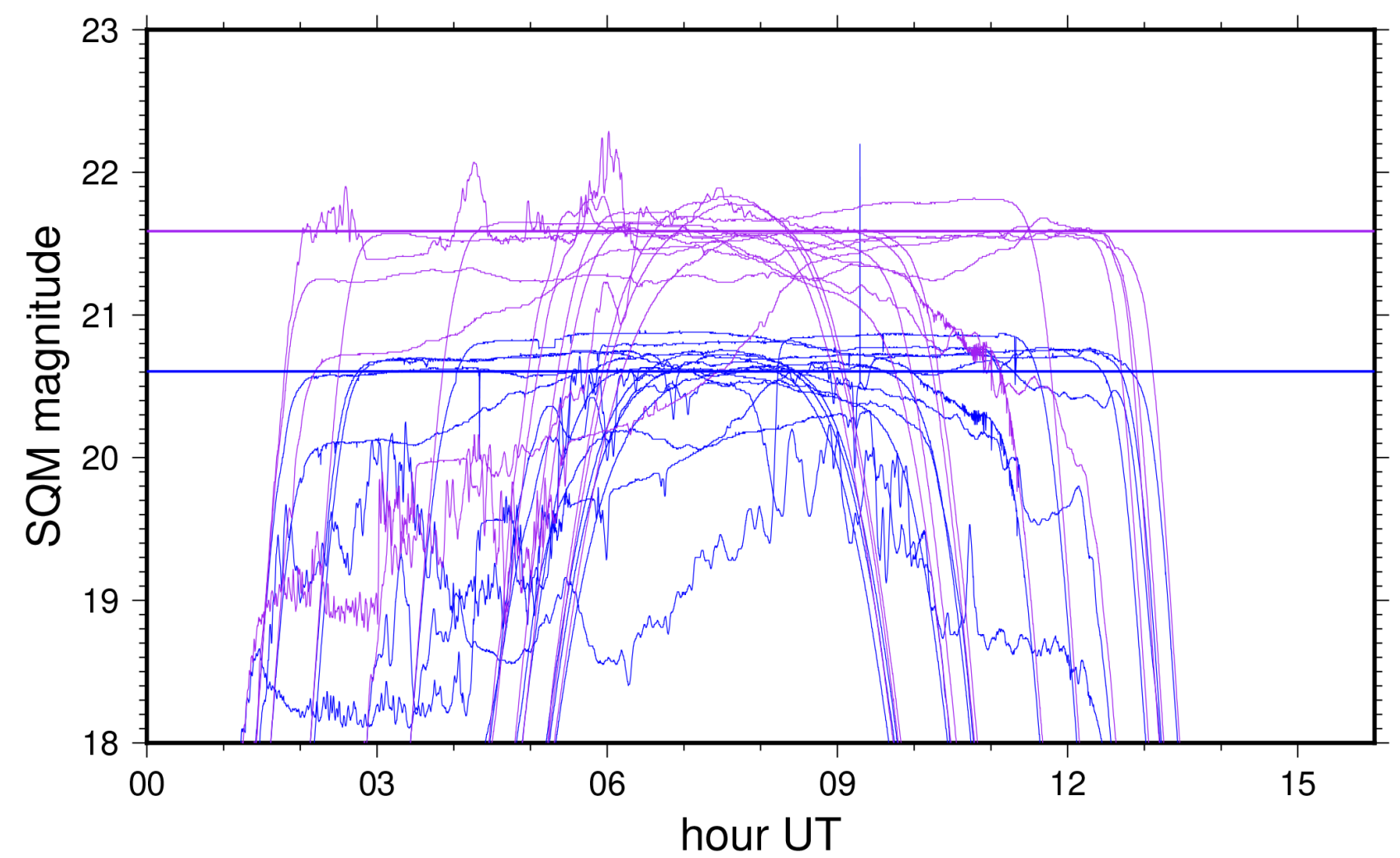

Figure 7. A subset of SQM sky brightness measurements from the AURT / AUGO observatory (in blue) and AUGSO observatory (in purple) taken between October 2017 and 2018. A clear distinction between the sites can be seen by overlying the data from 15 nights identified to have optimal dark sky conditions. The flat horizontal lines represent the average SQM sky brightness magnitude detected at the sites. At AUGSO, average sky brightness magnitude is 21.6, while at AURT / AUGSO, it is 20.6, a full magnitude lower. SQM measurements were taken within the span of time in the data when sky conditions were dark and clear, free from clouds, moon or aurora.

collaborative teaching and research opportunities. Recently we have partnered with UNC's Skynet group to host their robotic telescope at our AUGSO auroral observatory, which is an exceptional site due to its high-quality facilities and pristine dark-sky conditions. Although we cannot at this point see an expected brightening of the night sky over AURT due to insufficient data and the many variables at hand affecting dark sky conditions, we see a 1 magnitude difference in visual magnitude between the AUGSO and AURT sites. From the data we see that AUGSO is a remarkably dark site. As we continue to gather SQM data at both locations, we expect we will see a trend in light pollution. On the other hand, if dark sky conditions remain stable at both sites, this will point to effective light pollution mitigation practices being followed at the local level, which we would very much like to see.

\section{Acknowledgments}

We would like to thank the following groups and agencies for their part in supporting the development of AURT robotic telescope and Athabasca University's auroral observatories: Athabasca University Centre for Science and the Athabasca University Academic Research Committee; for their support of the first steps in developing robotic telescopes at AU. The Canada Foundation for Innova- 
tion (CFI); for supporting construction of AUGSO and AUGSO observatories. The Natural Sciences and Engineering Research Council of Canada (NSERC) for ongoing funding of Martin Connor's research. Additional thanks to Dan Reichart, Joshua Haislip and the Skynet Robotic Telescope Network, National Science Foundation grants: MRI-R2 0959447 , AAG 1009052,1211782, and 1517030, ISE 1223235, TUES 1245383, and STEM+C 1640131. Special thanks to Ian Shelton, Rob Lerner and others who played a role in establishing robotic telescope astronomy at Athabasca University.

\section{References}

Caton, D. (2018). Installing and Running Telescopes on Skynet for Research and Instruction. RTSRE, 1(1):137-143.

Childers, G., Watson, K., Jones, M. G., Williamson, K., and Hoette, V. (2015). TOUCHING THE STARS. Science Scope, 38(9):20.

Cinzano, P. (2005). Night sky photometry with sky quality meter. ISTIL Int. Rep, 9:1-14.

Connors, M. (2003). A decade's progress in distance education astronomy. Journal of the Royal Astronomical Society of Canada, 97(5):218-222.

Connors, M., Al-Shamali, F., Munyikwa, K., Schofield, I., and Bredeson, C. (2019). Three Decades of Distance Education Astronomy at Athabasca University. Robotic Telescope. RTSRE Proceedings, 2(1).

Connors, M., Bolton, D., and Doktor, I. (2016). Algolcam: Low Cost Sky Scanning with Modern Technology. In American Astronomical Society Meeting Abstracts \#227, volume 227 of American Astronomical Society Meeting Abstracts, page 427.05 .

Connors, M., Wiegert, P., Mainzer, A., Martin, B., and Veillet, C. (2014). The Discovery of Earth's Trojan Asteroid. , 108:54.

Connors, M., Wiegert, P., and Veillet, C. (2011). Earth's Trojan asteroid. , 475:481-483.
Guyon, O., Walawender, J., Jovanovic, N., Butterfield, M., Gee, W. T., and Mery, R. (2014). The PANOPTES project: discovering exoplanets with low-cost digital cameras. In Ground-based and Airborne Telescopes $V$, volume 9145 of , page 91453V.

Kyba, C. C., Tong, K. P., Bennie, J., Birriel, I., Birriel, J. J., Cool, A., Danielsen, A., Davies, T. W., Peter, N., Edwards, W., et al. (2015). Worldwide variations in artificial skyglow. Scientific reports, 5:8409.

Lang, D., Hogg, D. W., Mierle, K., Blanton, M., and Roweis, S. (2010). Astrometry.net: Blind astrometric calibration of arbitrary astronomical images. , 137:1782-2800. arXiv:0910.2233.

Langill, P. and George, B. (2017). Quantifying and Monitoring Darkness over the RAO. JRASC, 111(2):47.

Martin, B. and Connors, M. (2008). A One-meter Robotic Telescope for Western Canada.

Reichart, D. E. (2006). UNC-Chapel Hill's GammaRay Burst Follow-up Program. Society for Astronomical Sciences Annual Symposium, 25:39.

Sakaguchi, K., Shiokawa, K., Miyoshi, Y., and Connors, M. (2015). Isolated proton auroras and Pc1/EMIC waves at subauroral latitudes. Auroral dynamics and space weather, 215:59.

Smith, A. B., Caton, D. B., and Hawkins, R. L. (2016). Implementation and Operation of a Robotic Telescope on Skynet. , 128(5):055002. 